\title{
The childhood arthritis \& rheumatology research alliance network registry: demographics and characteristics of the initial 6-month cohort
}

\author{
Marc D Natter ${ }^{1 *}$, Jane R Winsor ${ }^{3}$, Kathleen A Fox ${ }^{3}$, Norman T llowite ${ }^{2}$, Kenneth D Mandl ${ }^{1}$, Kelly L Mieszkalski ${ }^{4}$, \\ Christy I Sandborg ${ }^{6}$, John S Sundy ${ }^{4}$, Carol A Wallace ${ }^{5}$, Laura E Schanberg ${ }^{4}$, CARRAnet Investigators Group ${ }^{7}$
}

From 2011 Pediatric Rheumatology Symposium sponsored by the American College of Rheumatology Miami, FL, USA. 2-5 June 2011

\section{Purpose}

In 2009, the Childhood Arthritis and Rheumatology Research Alliance (CARRA) established a longitudinal multi-center, multiple disease U.S. national registry (CARRAnet) for pediatric rheumatology with the intent of providing 60 participating clinical sites a new framework to drive observational clinical research and evidence-based care. CARRAnet seeks to enroll up to 20,000 subjects with childhood-onset rheumatic disease and twice yearly follow-up. We report baseline characteristics of the initial 6-month enrollment cohort; disease-specific results are reported separately.

\section{Methods}

Enrollment commenced 5/29/2010 with data available through 12/28/10. Inclusion criteria comprised 1 of 8 categories of defined rheumatic disease with onset before the $16^{\text {th }}$ birthday in subjects $<=21$ years (localized scleroderma, juvenile dermatomyositis, juvenile idiopathic arthritis, juvenile primary fibromyalgia syndrome, SLE or mixed connective tissue disease, sarcoidosis, systemic sclerosis, and vasculitis). A common baseline data set and 1 disease-specific data set was completed on each participant by interview and chart review. Data cleaning and analysis employed Microsoft Excel and Access (Microsoft Corp), SAS (SAS Institute), and R (R Foundation for Statistical Computing).

\section{Results}

1638 subjects were enrolled from 27 centers throughout the US. The analysis cohort reflected 1371 subjects,

${ }^{1}$ Children's Hospital Boston, Boston, MA, USA

Full list of author information is available at the end of the article
Table 1 Summary characteristics of CARRAnet initial cohort

\begin{tabular}{ll}
\hline Demographic measures & N (\%) \\
\hline Total participants studied & 1371 \\
Female gender & $1024(75 \%)$ \\
White/caucasian & $1187(87 \%)$ \\
Age at onset of symptoms, mean in years & 7.2 \\
Age at baseline visit, mean in years & 11.8 \\
Black or African American & $127(9 \%)$ \\
Asian & $45(3 \%)$ \\
American Indian or Alaska Native & $30(2 \%)$ \\
Native Hawaiian or Pacific Islander & $9(<1 \%)$ \\
Hispanic ethnicity & $150(11 \%)$ \\
\hline
\end{tabular}

\begin{tabular}{ll}
\hline Primary rheuamtic diagnosis & $1051(77 \%)$ \\
Juveline idiopathic arthritis & $109(8 \%)$ \\
Juveline dematophyositis & $100(7 \%)$ \\
Systemic lupus erythematosus & $43(3 \%)$ \\
Localized scleroderma & $23(2 \%)$ \\
Mixed connective tissue disease & $23(2 \%)$ \\
Vasculitis & $9(<1 \%)$ \\
Systemic sclerosis & $8(<1 \%)$ \\
Sarcoidosis & $6(<1 \%)$ \\
Juveline primary fibromyalgia syndrome & Mean \& (Range) \\
\hline Growth parameters & $0.28(-5.3-4.7)$ \\
Weight Z-score & $-0.14(-19.7-9.4)$ \\
Height Z-score & $0.38(-5.0-4.1)$ \\
BMI Z-score & Mean \& (Range) \\
\hline Assessments (scale: best -> worst) & $1.6(0-10)$ \\
Physician global assess disease activity (0 - 10) & $2.3(0-10)$ \\
Subject global assess disease activity (0 - 10) & $2.4(0-10)$ \\
Subject pain score (0 - 10) & $0.33(0-3)$ \\
CHAQ (0 - 3) & $96 \%$ \\
HRQOL - Good, very good, excellent (\%) &
\end{tabular}


Table 1 Summary characteristics of CARRAnet initial cohort (Continued)

\begin{tabular}{ll}
\hline HRQOL - Very poor, poor (\%) & $3 \%$ \\
\hline Family history (first degree relative) of & N (\%) \\
Psoriasis & $82(6 \%)$ \\
Rheumatoid arthritis & $71(5 \%)$ \\
Autoimmune throiditis & $62(5 \%)$ \\
Fibromyalgia & $61(4 \%)$ \\
Juvenile idiopathic arthritis & $55(4 \%)$ \\
Diabetes type I & $35(3 \%)$ \\
Inflammatory bowel disease & $31(2 \%)$ \\
Systemic lupus erthematosus & $28(2 \%)$ \\
Spondyloarthritis or ankyl. spondylitis & $16(1 \%)$ \\
Multiple sclerosis & $13(1 \%)$ \\
Celiac disease & $10(1 \%)$ \\
Uveitis & $5(<1 \%)$ \\
\hline Medication use & $\mathbf{N ~ ( \% )}$ \\
Steroids (ever) & $1010(74 \%)$ \\
Steroids (longterm daily, ever) & $644(47 \%)$ \\
Steroids (IV pulse, ever) & $212(15 \%)$ \\
Steroids (IA, ever) & $529(39 \%)$ \\
Biologics (ever) & $560(41 \%)$ \\
Biologics (current) & $429(31 \%)$ \\
TNF- $\alpha$ Blockers (current) & $338(25 \%)$ \\
DMARDs (ever) & $1107(81 \%)$ \\
DMARDs (current) & $1021(75 \%)$ \\
Methotrexate (current) & $620(45 \%)$ \\
NSAIDs (current) & $625(46 \%)$ \\
Opioids (current) & $17(1 \%)$ \\
\hline &
\end{tabular}

predominantly JIA; 63 variables were collected for the shared baseline form with summary statistics presented in the figures. The population reported overall good to excellent health by patient and physician report: $96 \%$ with mean HRQOL good to excellent; physician mean global assessment of disease activity (PGAS) 1.6 (0-10 scale). PGAS correlated with subject reports (CHAQ, subject global, subject pain scores - Pearson corr 0.33, $0.39,0.42$ respectively). Medication use was prevalent, including $74 \%$ ever on steroids, $41 \%$ ever on biologics, and $31 \%$ currently on biologics. Growth was within normal on average, but exhibited wide deviation (-5.3>weight $Z>4.7,-19.7>$ height $Z>9.4)$. A similarly wide range was seen on both objective and subjective measures, identifying probable subpopulations with high disease activities.

\section{Conclusion}

The initial CARRAnet cohort reflects predominantly low disease activity with favorable self-reports. This is not a population study and issues of enrollment bias require further investigation. Despite the overall well-being of

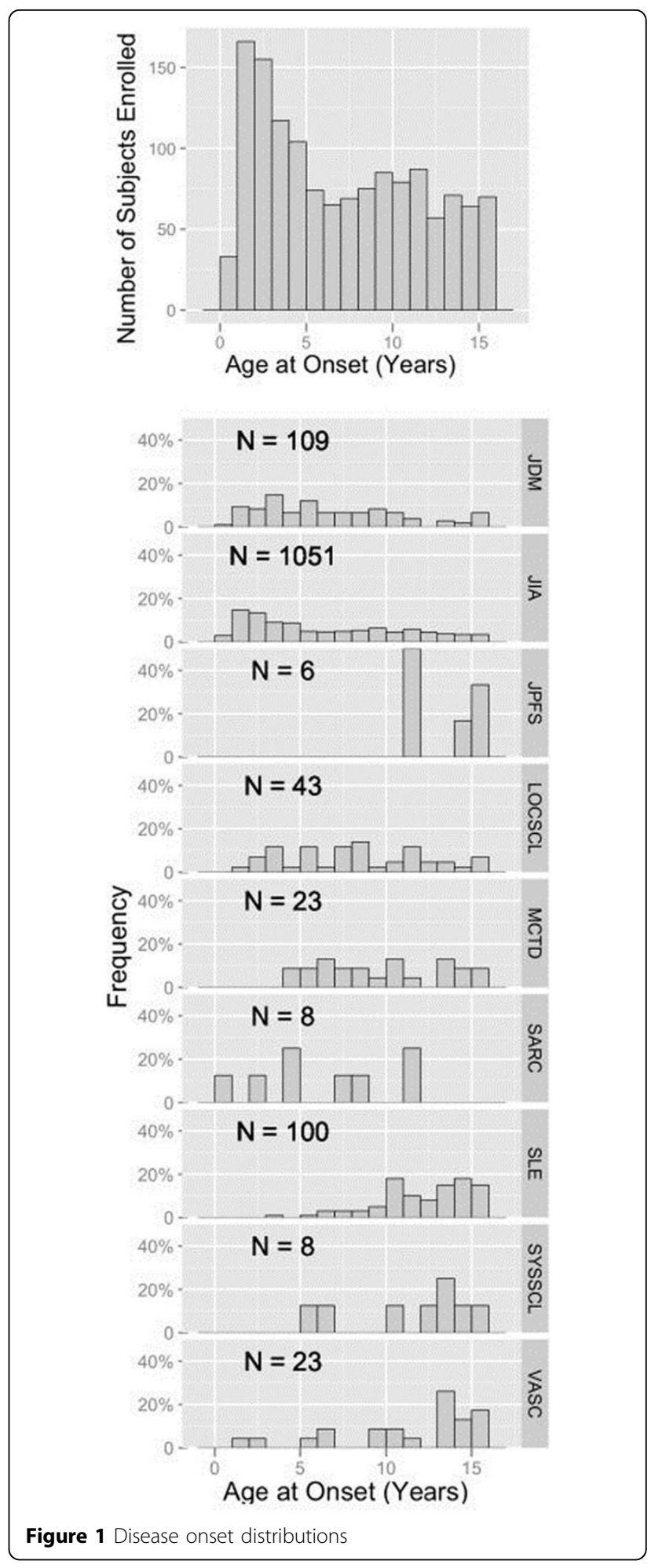

the population, the high use of steroids, biologics, and DMARDs, along with significant subpopulations concerning for high disease activity, are important areas of future focus. 


\section{Disclosure}

Marc D. Natter: None; Jane R. Winsor: None; Kathleen A. Fox: None; Norman T. Ilowite: None; Kenneth D. Mandl: None; Kelly L. Mieszkalski: None; Christy I. Sandborg: None; John S. Sundy: None; Carol A. Wallace: None; Laura E. Schanberg: None; CARRAnet Investigators Group: None.

\section{Author details}

${ }^{1}$ Children's Hospital Boston, Boston, MA, USA. ${ }^{2}$ Children's Hospital Montefiore, Bronx, NY, USA. ${ }^{3}$ Duke Clinical Research Institute, Durham, NC USA. ${ }^{4}$ Duke University Medical Center, Durham, NC, USA. ${ }^{5}$ Seattle Children's Hospital \& Regional Medicine, Seattle, WA, USA. ${ }^{6}$ Stanford Medical Center, Stanford, CA, USA. ${ }^{7}$ Stanford, CA, USA.

Published: 13 July 2012

\section{doi:10.1186/1546-0096-10-S1-A57}

Cite this article as: Natter et al:: The childhood arthritis \& rheumatology research alliance network registry: demographics and characteristics of the initial 6-month cohort. Pediatric Rheumatology 2012 10(Suppl 1):A57.

Submit your next manuscript to BioMed Central and take full advantage of:

- Convenient online submission

- Thorough peer review

- No space constraints or color figure charges

- Immediate publication on acceptance

- Inclusion in PubMed, CAS, Scopus and Google Scholar

- Research which is freely available for redistribution

Submit your manuscript at www.biomedcentral.com/submit 